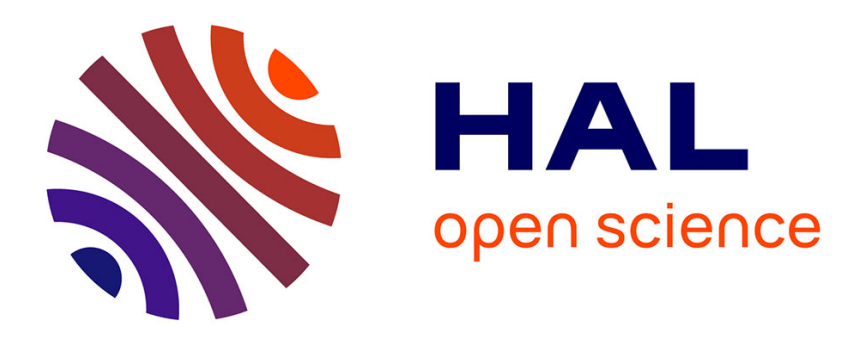

\title{
Une approche sémantique non prototypique de la construction transitive
}

\author{
Lise Hamelin, Dominique Legallois
}

\section{To cite this version:}

Lise Hamelin, Dominique Legallois. Une approche sémantique non prototypique de la construction transitive. Faits de langues, 2015. hal-03391784

\section{HAL Id: hal-03391784 \\ https://hal.science/hal-03391784}

Submitted on 21 Oct 2021

HAL is a multi-disciplinary open access archive for the deposit and dissemination of scientific research documents, whether they are published or not. The documents may come from teaching and research institutions in France or abroad, or from public or private research centers.
L'archive ouverte pluridisciplinaire HAL, est destinée au dépôt et à la diffusion de documents scientifiques de niveau recherche, publiés ou non, émanant des établissements d'enseignement et de recherche français ou étrangers, des laboratoires publics ou privés. 


\title{
Une approche sémantique non prototypique de la construction transitive
}

\author{
Lise Hamelin* et Dominique Legallois **
}

\section{INTRODUCTION}

Élaborée essentiellement dans le cadre de la linguistique générale et dans celui de la typologie, la notion de transitivité prototypique s'est imposée depuis les articles de Lakoff (1977) et Hopper et Thompson (1980), comme indispensable pour comprendre la motivation des schémas bi-actanciels dans les langues. Les paramètres conduisant à la définition de la prototypicité (ou de la haute transitivité) ont fait l'objet, depuis, de nombreux travaux, d'ailleurs relativement consensuels malgré des variantes mineures.

Sans minimiser l'importance de la notion de transitivité prototypique, l'approche présentée dans cet article propose une voie quelque peu différente, que trois points peuvent caractériser : premièrement, ce travail s'inscrit dans la perspective d'une sémantique des constructions grammaticales. Il a pour objectif de tester l'hypothèse selon laquelle un schéma syntaxique peut posséder, indépendamment du lexique, une signification. Le schéma étudié est donc le schéma transitif, noté par commodité NVN ; NVN correspond alors à la séquence formée d'un GN1 sujet, d'un verbe, et d'un GN2 complément d'objet' ${ }^{\text {. }}$

Deuxièmement, l'étude est empirique. Elle s'appuie sur un traitement statistique des usages de NVN, afin d'identifier dans un corpus oral, les verbes les plus attirés par cette structure. Ces verbes sont-ils les plus typiques de la transitivité sémantique? Il s'agira d'appliquer à la syntaxe et à la sémantique, des méthodes éprouvées en linguistique de corpus et en lexicologie.

Troisièmement, contrairement à beaucoup d'études, l'approche défendue considère que dans le cadre d'une analyse sémantique, les cas de transitivité syntaxique non prototypique ne doivent pas être traités comme des occurrences «dégradées» (par extension, par métaphore, etc.) de cas de transitivité prototypique. Autrement dit, la transitivité sémantique ne constitue pas le terminus a quo à partir duquel il est possible de comprendre les mécanismes de

\footnotetext{
*LDI, Université de Cergy-Pontoise, Courriel : lise.hamelin@u-cergy.fr

${ }^{* *}$ CRISCO, Université de Caen, Courriel : dominique.legallois@unicaen.fr

${ }^{1}$ L'étude porte essentiellement sur le français, et ne concerne pas les cas où le complément d'objet est une complétive.
} 
variation menant à la transitivité non prototypique. Au contraire, nous considérerons que, malgré l'hétérogénéité des classes verbales liées au schéma, la signification de NVN est suffisamment cohérente pour rendre compte de la diversité des cas.

L'article est organisé comme suit : la première partie rappelle la conception de la transitivité prototypique - les principaux paramètres qui la constituent, sa justification et sa pertinence pour la typologie, la psycholinguistique et la lexicologie.

La deuxième partie est consacrée à l'analyse d'un corpus oral. Par une étude statistique, il sera mis en évidence que les verbes les plus attirés par NVN ne sont pas ceux considérés comme typiques de la transitivité sémantique. L'analyse de quelques exemples montrera que l'on peut malgré tout observer un rapport constant dans les énoncés non typiques, entre le référent du sujet et celui de l'objet. Ce rapport, à notre connaissance, n'a jamais été mentionné par les études sur la transitivité.

La troisième partie montre qu'il s'avère possible de dégager une signification suffisamment cohérente pour appréhender toutes les occurrences de NVN. Les relations de conjonction et de disjonction (du sujet avec l'objet) constituent des notions sémantiques abstraites, mais fondamentales, jouant un rôle essentiel dans la signification des occurrences de NVN. Nous montrerons que cette signification s'applique également à la transitivité prototypique. Ces rapports de conjonction / disjonction du sujet avec l'objet seront enrichis par un autre type de relation : relation entre l'élément sujet ou objet et ce que nous nommerons l'espace subjectal ou objectal.

La quatrième et dernière partie est composée d'un ensemble de discussions dont l'objectif est à la fois d'éclaircir certains comportements de NVN que permet de dégager notre modèle, et d'apporter des arguments supplémentaires pour justifier la conception défendue ici. Ainsi, seront examinés des exemples de double lecture d'un même énoncé transitif : un énoncé peut parfois être interprété en privilégiant une lecture causale de l'événement exprimé (c'est l'affectation de l'objet qui est alors focalisée) ; parfois, cet énoncé peut être lu sans privilégier une quelconque causalité (c'est alors le rapport entre le sujet et l'objet qui est focalisé). Dans cette partie, nous montrerons également que les notions de conjonction et de disjonction - celles-là mêmes qui sont en œuvre dans la transitivité simple - sont également indispensables pour appréhender les significations des constructions trivalentes avec datif (par ex. prêter de l'argent à quelqu'un) et les constructions transitives simples avec objet obligatoirement pluriel (séparer les enfants).

La conclusion de l'article pointe à la fois les problèmes qui restent à traiter et les perspectives ouvertes par cette étude.

Plus que d'apporter des résultats fermes et définitifs, ce travail, au contraire, met en évidence des «conclusions» qui ne peuvent être, étant donnée la complexité du sujet, que provisoires. Ses deux ambitions principales sont: d'abord, de dégager un premier bilan sur le comportement de NVN et sur son statut de construction; ensuite, de donner au lecteur les éléments essentiels 
(empiriques, analytiques, théoriques) à partir desquels il pourra apprécier de façon critique l'approche défendue dans ces pages.

\section{LA TRANSITIVITÉ PROTOTYPIQUE}

\subsection{Prototypicité}

On rappellera rapidement la conception prototypique de la transitivité, qui a pour origine les articles de Lakoff (1977) et surtout Hopper et Thompson (1980). Nous reproduisons le tableau que François (2003) propose pour caractériser les traits essentiels de ce que Hopper et Thompson nomment la «haute transitivité» ${ }^{2}$.

\begin{tabular}{|c|c|}
\hline Catégorie grammaticale & Traits de transitivité prototypique \\
\hline $\begin{array}{l}\text { ACTANCE } \\
\text { Participants } \\
\text { Kinesis } \\
\text { Agentivité } \\
\text { Affectation de l'objet } \\
\text { caractère volontaire }\end{array}$ & $\begin{array}{l}\rightarrow \text { deux ou plus } \\
\rightarrow \text { action } \\
\rightarrow \text { hautement agentif } \\
\rightarrow \text { objet totalement affecté } \\
\rightarrow \text { volontaire }\end{array}$ \\
\hline $\begin{array}{l}\text { ASPECT } \\
\text { Aspect } \\
\text { Ponctualité }\end{array}$ & $\begin{array}{l}\rightarrow \text { télique (borné) } \\
\rightarrow \text { ponctuel }\end{array}$ \\
\hline $\begin{array}{l}\text { DETERMINATION NOMINALE } \\
\text { Individuation de l'objet }\end{array}$ & $\rightarrow$ hautement individualisé \\
\hline $\begin{array}{l}\text { ENONCIATION } \\
\text { Affirmation } \\
\text { Mode }\end{array}$ & $\begin{array}{l}\rightarrow \text { affirmative (polarité positive) } \\
\rightarrow \text { indicatif (realis) }\end{array}$ \\
\hline
\end{tabular}

Tableau 1 : Critères de haute transitivité selon Hopper et Thompson (1980) d'après François (2003)

Bien que la transitivité soit considérée comme multifactorielle, l'affectation (qui comprend également l'effectuation) est le trait fondamental par lequel la transitivité sémantique est généralement définie ${ }^{3}$. Les modes d'affectation ont fait l'objet d'une classification par Desclés (1998) : objet créé (il a construit une maison), objet détruit (elle a cassé un verre), changement physique de l'objet (elle découpe le poulet), changement de position de l'objet (il déplace le lit), modification de l'objet avec un instrument (il fusille les prisonniers), changement de propriété de l'objet (il peint le salon), changement interne (il réforme le monastère).

Les exemples (repris de Desclés) élaborent le schème de transitivité sémantique (STS), dont rend compte la figure ci-dessous :

\footnotetext{
${ }^{2}$ Cf. aussi Larjavaara (2000). Pour une discussion et une critique des propriétés dégagées par Hopper et Thompson, cf. Tsunoda (1985).

${ }^{3}$ Cependant pour Naess (2007) c'est le différentiel de participation entre sujet et objet, qui constitue le paramètre fondamental de la transitivité.
} 


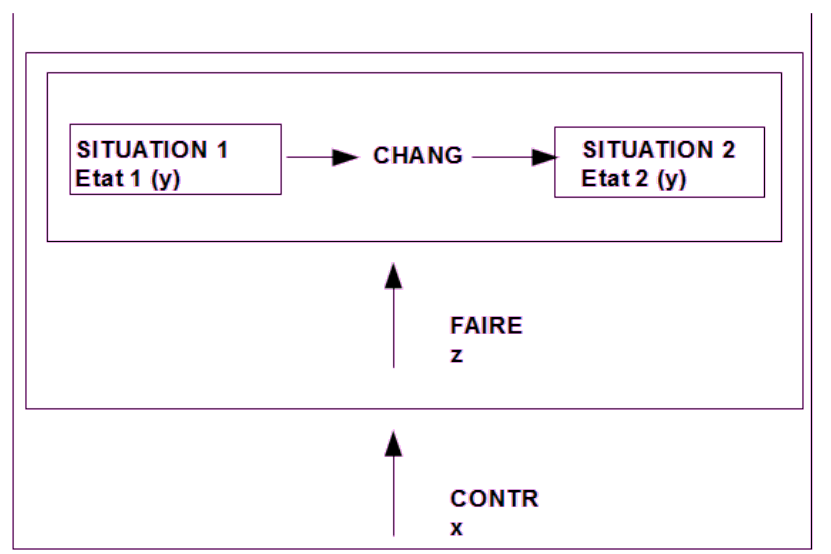

Figure 1 : Représentation du STS selon Desclés 1998

$\mathrm{X}$ est le contrôleur d'une effectuation opérée par Z. X et $\mathrm{Z}$ peuvent être identiques (Paul ouvre la porte) ou non (Paul ouvre la porte avec une clé). Le schéma cinématique relatif au changement (CHANG) pour y (l'objet) de SIT1 à SIT2, est emboîté dans le schéma d'effectuation (FAIRE), lui-même emboîté dans le schéma de contrôle (CONTR) - le contrôle se définissant par «la capacité de déclencher et d'interrompre une action» (Desclés 1990 : 225). Les mécanismes d'extension à des verbes non typiques (que nous notons à présent NON-CHANG par opposition aux verbes CHANG) doivent donc être expliqués à partir de ce schéma ${ }^{4}$. Plusieurs auteurs (dont Givon 1984 et 1989, Langacker 1990, Desclés 1998) ont donc entrepris d'expliquer ces mécanismes d'extension de CHANG à NON-CHANG par une dégradation de l'agentivité du sujet. Ainsi, pour

(1) he saw the moutain /she knew the answer / he heard the song / she feared him / he wanted a sandwich / she remembered the conversation

Givon constate que :

\footnotetext{
${ }^{4} \mathrm{La}$ distinction entre verbes prototypiques et verbes non prototypiques n'est qu'une réminiscence de la distinction entre verbes d'action réelle et verbes d'action intentionnelle formulée par Arnault et Lancelot : «On appelle proprement actifs, ceux [|les verbes] qui signifient une action à laquelle est opposée une passion, comme battre, être battu; aimer, être aimé ; soit que ces actions se terminent à un sujet, ce qu'on appelle action réelle, comme battre, rompre, tuer, noircir, etc. soit qu'elle se termine seulement à un objet, ce qu'on appelle action intentionnelle, comme aimer, connaître, voir» (Arnault et Lancelot, 1973/1676 : 100). Sujet et objet n'ont pas ici leur acception grammaticale. Aussi faut-il traduire sujet par objet et objet par sujet pour ne pas faire de contresens de lecture.
} 
English seems to be relaxing an important part of the definition of «agent», moving from the stricter condition of «having volition, control and decision-to-act» to a weaker condition of «having conscious mental activity». Such extension is again an instance of generalization or semantic bleaching, based on the fact that both volition and decision-to-act are themselves mental activities (Givon, 1989:62).

\subsection{Justification typologique}

La conception prototypique de la transitivité a essentiellement été défendue dans le champ de la typologie ${ }^{5}$. Ainsi Lazard $(1998,2008)$ montre-t-il que l'action prototypique universelle (se définissant comme «action réelle et complète exercée volontairement par un agent humain sur un patient bien individué qui en est effectivement affecté» $\left.{ }^{6}\right)$ ne peut être exprimée, quelle que soit la langue, que par une CBM (construction bi-actancielle majeure). Dans certaines langues comme l'avar, le russe, la CBM serait même exclusivement dédiée à l'expression de l'action prototypique. Un exemple proposé par Creissels (2013) illustre le phénomène : en Mandinka, langue mandingue du Sénégal, le verbe tee signifie soit «couper», soit «traverser», la relation entre les deux acceptions étant suffisamment évidente pour écarter toute homonymie. Cependant, lorsque tee signifie «couper» (emploi CHANG), seule la construction directe est possible ; lorsque tee signifie «traverser» (emploi NONCHANG), la construction directe et la construction oblique sont non seulement possibles, mais synonymes :
(2) Mǒo-lu ka báa tee. personne.D-PL I NACP.POS fleuve.D traverser 'les gens traversent le fleuve'

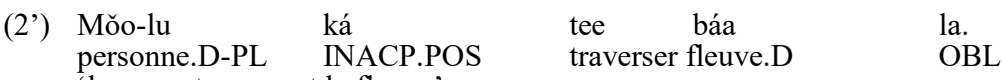
'les gens traversent le fleuve'

(3) Mǒo-lu ká ñantáy-o tee. personne.D-PL INACP.POS paille-D couper 'les gens coupent de la paille'

(3’) *Mǒo-lu ká tee ñantán-o la. personne.D-PL INACP.POS couper paille-D OBL

\subsection{Justification psycholinguistique}

L'expérience menée par Ibbotson et al. (2012) a consisté à mettre en évidence une différence notable de traitement de la transitivité sémantique entre enfants (entre 3,9 et 4,9 ans) et adultes. On présente à chaque population un ensemble d'énoncés transitifs dont aucun ne peut être considéré comme prototypique ;

\footnotetext{
${ }^{5}$ Cf. Naess (2007).

${ }^{6}$ De même, dans son étude sur la transitivité portant sur 35 langues, Haspelmath (à par.) considère les verbes qui, pour chaque langue, se construisent de la même façon que celui qui exprime l'action de «couper» (BREAK).
} 
après un certain laps de temps, on demande aux sujets de reconnaître parmi une série d'énoncés quels sont ceux qui ont été présentés dans la phase 1. Cette deuxième série est composée effectivement de certains des énoncés de la phase 1 , mais aussi d'énoncés non présentés préalablement, et pouvant être caractérisés de transitivement prototypiques. Les adultes ont une tendance marquée à «reconnaître» ces énoncés prototypiques, comme s'ils avaient été présentés lors de la phase 1 ; les enfants ont tendance à ne pas faire cette erreur. L'explication de l'erreur des adultes peut être celle-ci : la catégorie de la transitivité est organisée autour d'un prototypique qui permettrait de donner une cohérence à l'ensemble des énoncés non typiques; autrement dit, c'est par rapport à une dimension prototypique convoquée par les adultes, que les énoncés «déviants» sont appréhendés, non pas de façon isolée, mais comme des membres non typiques d'une même catégorie. Les enfants n'auraient pas encore acquis cette organisation de la catégorie.

\subsection{Justification lexicologique}

Les partisans de l'approche prototypique oublient généralement un argument qui joue en faveur de leur thèse : la productivité lexicale. L'examen d'un ensemble de 3000 verbes français pouvant être employés transitivement, montre que moins de $10 \%$ des lexèmes ne peuvent pas être considérés comme prototypiques (par ex. admettre, accueillir, ignorer, observer, suivre...). Ces verbes NON-CHANG semblent en revanche beaucoup plus communs - c'est-àdire fréquents - que l'ensemble des verbes présentant un plus grand degré de transitivité (CHANG). Dans ce dernier ensemble, en effet, figurent un grand nombre de verbes relativement rares, en raison de la spécialisation du procès qu'ils expriment (par ex. nomadiser, praliner, radioscoper, etc.). L'explication d'un tel déséquilibre entre classe NON-CHANG et classe CHANG est simple : «l'industrialisation», ou si on veut, le changement que nous faisons subir à notre environnement, est un trait de notre civilisation dont le besoin d'expressivité doit être comblé - d'où le grand nombre de verbes sémantiquement transitifs, et les «solutions» morphologiques par les affixes -dé(s) (désodoriser), -ifier (modifier), -iser (municipaliser).

\subsection{Bilan}

Le modèle prototypique possède indiscutablement une validité ; pourtant, certains auteurs s'inscrivant dans une perspective empirique (Thompson et Hopper 2001, Hagège 2006, Vázquez Rozas 2007) ont souligné un problème important qui a été très peu discuté : la conversation, qui constitue le genre premier et fondamental par excellence, use d'un taux d'énoncés de haute transitivité extrêmement faible, tandis que les cas non typiques sont nombreux. Cette réalité ne constitue pas un argument pour nier toute validité à la prototypicité : un prototype peut être moins fréquent que des cas non typiques. La prototypicité est une question de saillance plutôt que de fréquence. La critique porte plutôt sur la nécessité d'expliquer ce qui est relativement ordinaire dans 
l'expérience discursive par ce qui est plus exceptionnel. Le statut théorique de la notion de transitivité prototypique, s'il est légitime en typologie, en lexicologie, voire en psycholinguistique, l'est-il toujours en sémantique?

La section suivante rend compte d'une analyse sur corpus du schéma NVN. Les données constitueront une base sur laquelle s'appuiera une conception sémantique non extensionnelle de la transitivité : autrement dit, sera défendue l'hypothèse que cas non typiques et typiques répondent à un même invariant sémantique, et qu'il n'y a pas lieu de faire dériver le premier type de cas du second.

\section{NVN À L'EPREUVE DES DONNÉES ATTESTÉES}

\subsection{Description du corpus}

Le corpus choisi (CFPP) est un sous-ensemble de 68152 mots du corpus CFPP $2000^{7}$ constitué d'interviews de Parisiens sur leur mode de vie, leur environnement, leur quartier. CFPP comporte 966 occurrences du schéma NVN ${ }^{8}$ et 155 lexèmes intègrent ce schéma. L'analyse consiste en un comptage en termes de fréquence des verbes, mais surtout en un calcul d'attirance des verbes pour le schéma, en adoptant la méthode statistique des collostructions mise au point par Stefanowitsch, et Gries (2003).

Le tableau suivant présente, par ordre, les 50 premiers verbes. Sont donnés : le lexème verbal, la fréquence du verbe dans le schéma, la fréquence attendue (théorique), et la «force»d'attraction, c'est-à-dire l'écart, calculé par le loglikelihood, entre la fréquence théorique et la fréquence observée.

\begin{tabular}{|l|l|l|l|l|}
\hline Verbe & $\begin{array}{c}\text { Frq totale } \\
\text { du verbe }\end{array}$ & $\begin{array}{c}\text { Frq du verbe } \\
\text { dans NVN }\end{array}$ & Frq théorique & $\begin{array}{c}\text { Force d'attirance du } \\
\text { verbe pour NVN }\end{array}$ \\
\hline avoir & 2039 & 305 & 28.901 & 1016.956 \\
\hline faire & 412 & 141 & 5.84 & 698.86 \\
\hline connaître & 131 & 52 & 1.857 & 271.606 \\
\hline prendre & 71 & 41 & 1.006 & 254.865 \\
\hline voir & 235 & 49 & 3.331 & 184.035 \\
\hline acheter & 45 & 21 & 0.638 & 117.707 \\
\hline marquer & 18 & 14 & 0.255 & 100.421 \\
\hline entendre & 37 & 16 & 0.524 & 86.44 \\
\hline aimer & 74 & 15 & 1.049 & 54.969 \\
\hline inviter & 12 & 8 & 0.17 & 53.004 \\
\hline
\end{tabular}

\footnotetext{
${ }^{7}$ http://cfpp2000.univ-paris3.fr/ ; cf. Branca-Rosoff et al (2012).

8 Nous avons également pris en compte les cas de pronominalisation de l'objet, par clitique ou par pronom relatif.
} 


\begin{tabular}{|c|c|c|c|c|}
\hline garder & 19 & 9 & 0.269 & 50.693 \\
\hline poser & 20 & 9 & 0.283 & 49.483 \\
\hline traverser & 14 & 8 & 0.198 & 49.215 \\
\hline attirer & 9 & 6 & 0.128 & 39.741 \\
\hline pousser & 9 & 6 & 0.128 & 39.741 \\
\hline chercher & 24 & 8 & 0.34 & 38.067 \\
\hline trouver & 115 & 14 & 1.63 & 37.036 \\
\hline esquinter & 4 & 4 & 0.057 & 34.067 \\
\hline regarder & 13 & 6 & 0.184 & 33.367 \\
\hline retrouver & 27 & 7 & 0.383 & 29.303 \\
\hline apprécier & 5 & 4 & 0.071 & 29.091 \\
\hline porter & 5 & 4 & 0.071 & 29.091 \\
\hline manger & 12 & 5 & 0.17 & 26.487 \\
\hline boire & 3 & 3 & 0.043 & 25.547 \\
\hline concerner & 3 & 3 & 0.043 & 25.547 \\
\hline intéresser & 7 & 4 & 0.099 & 24.592 \\
\hline rencontrer & 7 & 4 & 0.099 & 24.592 \\
\hline vendre & 7 & 4 & 0.099 & 24.592 \\
\hline louer & 15 & 5 & 0.213 & 23.778 \\
\hline perdre & 8 & 4 & 0.113 & 23.09 \\
\hline créer & 9 & 4 & 0.128 & 21.844 \\
\hline tenir & 18 & 5 & 0.255 & 21.688 \\
\hline fermer & 10 & 4 & 0.142 & 20.777 \\
\hline frapper & 13 & 4 & 0.184 & 18.275 \\
\hline acquérir & 2 & 2 & 0.028 & 17.029 \\
\hline déchaîner & 2 & 2 & 0.028 & 17.029 \\
\hline émouvoir & 2 & 2 & 0.028 & 17.029 \\
\hline jeter & 2 & 2 & 0.028 & 17.029 \\
\hline prolonger & 2 & 2 & 0.028 & 17.029 \\
\hline rayer & 2 & 2 & 0.028 & 17.029 \\
\hline réserver & 2 & 2 & 0.028 & 17.029 \\
\hline choisir & 7 & 3 & 0.099 & 16.1 \\
\hline
\end{tabular}




\begin{tabular}{|l|l|l|l|l|}
\hline ouvrir & 17 & 4 & 0.241 & 15.886 \\
\hline sentir & 19 & 4 & 0.269 & 14.937 \\
\hline suivre & 9 & 3 & 0.128 & 14.261 \\
\hline découvrir & 22 & 4 & 0.312 & 13.717 \\
\hline agrandir & 3 & 2 & 0.043 & 13.239 \\
\hline détester & 3 & 2 & 0.043 & 13.239 \\
\hline embêter & 3 & 2 & 0.043 & 13.239 \\
\hline fréquenter & 3 & 2 & 0.043 & 13.239 \\
\hline
\end{tabular}

Tableau 2 : Attirance des (50 premiers) verbes pour NVN dans le corpus CFPP

\subsection{Commentaires}

Ce tableau est nécessairement synthétique et ne rend pas compte a priori de facteurs tels que la polysémie des verbes, ou les types d'objets. Néanmoins, il donne un état des lieux exploitables des relations entre lexèmes verbaux et NVN dans le corpus. De plus, le retour au texte permet d'observer certaines particularités. Ainsi, le verbe faire est très majoritairement (105/141) employé comme verbe support, donc NON-CHANG : faire $d u$ vélo, faire les courses, faire une grande école, faire du sport, faire le ménage, etc. Il est beaucoup moins employé comme verbe CHANG (39/141): faire un duplex, faire un appartement, faire des graffitis, faire un ourlet, etc. Le verbe prendre est essentiellement support (prendre le café, mais surtout prendre + moyen de transport - taxi, métro, bus, etc.). Le verbe avoir, quant à lui, n'est généralement pas classé parmi les verbes transitifs. Il reste pourtant le verbe à la fois le plus attiré par NVN et le plus fréquent.

Le tableau montre sans ambiguïté que :

- les verbes CHANG ne sont pas les plus attirés par la construction : seuls faire-CHANG et marquer (dans l'expression ça m'a marqué) sont présents dans les 10 premiers rangs ;

-les verbes CHANG ont une fréquence modeste (par ex. esquinter, créer, agrandir, etc) ;

- les verbes NON-CHANG sont les plus attirés par NVN (avoir, connaître, voir, aimer, garder, etc.) ;

- les verbes NON-CHANG sont également les plus fréquents.

Par ailleurs, la propriété CHANG ou NON-CHANG de certains verbes est difficile à interpréter hors contexte (par ex. inviter, vendre, acheter). Le retour au corpus ne permet pas toujours de trancher entre une lecture cinématique (l'objet passe d'une SIT1 à une SIT2) ou stative (NON-CHANG) :

(4) mon fils aîné comme c'était l'aîné il me dit "est-ce que j'peux inviter mes copains enfin la clas- mes mes amis à une boum $+\mathrm{j}$ 'invite toute la classe à une boum 
(5) en fait c'est par mon grand-père qui avait acheté un appartement près du boulevard des Invalides

L'objet passe-t-il, respectivement, du statut de non-invité à celui d'invité, ou de «non acheté» à «acheté»? Ou bien doit-on considérer qu'aucune transformation n'est opérée dans ces exemples ? On verra que cette ambiguïté, loin de poser problème, est en fait constitutive du schéma NVN.

\subsection{Première considération sémantique de $N V N$}

Une linguistique fondée sur l'usage (usage-based) se doit de prendre en compte le type de données que présente le tableau 2 : la fréquence d'usage des verbes NON-CHANG ne peut pas ne pas être sans incidence sur le schéma NVN. Deux attitudes sont alors possibles : soit conclure à l'hétérogénéité de ce schéma qu'intègrent des classes de verbes différentes, et ne voir dans ces faits qu'un ensemble d'irrégularités - et dans ce cas, NVN ne peut être considéré comme une construction; soit procéder à une analyse qui tente d'associer au schéma une signification cohérente. C'est cette deuxième attitude qui est choisie ici.

Soit les 5 énoncés suivants, extraits du corpus :

(6) Y'en avait toujours un qui avait une voiture

(7) vous remontez la rue vous regardez la boutique

(8) et vous avez accepté les copains à la maison?

(9) j'aime bien cet environnement architectural très harmonieux avec des larges avenues

(10) c'était toujours vraiment un parcours du combattant hein + réserver un court le matin dès sept heures

Malgré des différences, ces énoncés sont motivés par l'expression d'une même relation abstraite entre le sujet et l'objet, cette relation prenant des formes très variées. Dans ces exemples, le référent du sujet entretient un rapport fondamental avec le référent de l'objet : le procès dans lequel est engagé le sujet aboutit à un «contact» (parfois concret, parfois abstrait, parfois effectif, parfois anticipé), un lien, une connexion avec l'objet. Ainsi, 6 exprime une relation de propriété : le sujet possède l'objet. La possession constitue un contact ou un lien - mais aussi, évidemment, bien plus que cela - avec l'objet'. En 7, même si la boutique n'est pas un objet visé pour ses propriétés intrinsèques, elle n'en est pas moins un repère avec lequel le sujet doit entrer en contact (visuel) pour pouvoir s'orienter. Il en est de même pour $8:$ accepter exprime un procès portant sur une attitude après délibération (refus/acceptation) relative au contact (social) entre «vous» et les copains. En 9, la relation avec l'objet est affective ${ }^{10}$. Les verbes affectifs ont

\footnotetext{
${ }^{9}$ Cf. Blinkenberg (1960) sur les verbes transitifs exprimant la possession ou des notions proches.

${ }^{10}$ Cf. Condamines (2013) sur la façon dont l'affectivité favorise la transitivation de compléments circonstanciels.
} 
pour fonction d'exprimer un lien d'attirance (ou de répulsion) entre sujets et objets. On pourrait parler de contact affectif entre le sujet et l'objet. En 10, réserver précise un contact qui n'est pas naturellement effectif, et qu'il convient donc de programmer. Son synonyme retenir exprime sans doute de façon plus claire encore l'idée que le sujet s'approprie pour un temps l'objet.

Ces quelques exemples peuvent encore paraitre insuffisants pour justifier le rapport invariable entre sujet et objet. Aussi, la partie suivante a pour objectif de préciser ce rapport, ainsi que le modèle sémantique dans lequel notre analyse prend place.

\section{SÉMANTIQUE DE $N V N$}

\subsection{Conjonction, disjonction, jonction}

Nous désignons la relation entre le sujet et l'objet par le terme de conjonction $^{11}$; cette relation est notée $\mathbf{X} \boldsymbol{\Lambda} \mathbf{Z}$ et se lit : le référent du sujet $(\mathrm{X})$ est en conjonction $(\Lambda)$ avec le référent de l'objet $(Z)$. La relation de conjonction est toujours asymétrique, orientée de $\mathrm{X}$ vers $\mathrm{Z}$. Le terme de conjonction vaut pour un ensemble de relations dont on voit bien qu'elles partagent le même fondement : avoir, avoir à sa disposition, avoir de l'attirance pour, détenir, tenir, saisir, toucher, être en contact avec, s'approprier, posséder, prendre, etc. Aussi, la relation de conjonction fait sens dans des domaines d'expérience différents; parmi ceux-ci :

\section{le domaine social}

(11) si j' vais à Ecole Militaire j' rencontre toujours des amis à moi

La rencontre est évidemment un événement où des personnes entrent en contact ;

le domaine perceptuel

(12) dans l'quartier vous entendez des merles

Toute perception - quelle que soit la modalité - élabore un contact du sujet percevant avec l'objet perçu; autrement dit, percevoir, c'est saisir ou prendre connaissance par les sens.

\section{le domaine affectif}

(13) moi j'avais faim j'ai pris des chichis j'adore les chichis

L'affectivité concerne l'attirance que l'on a pour un objet, ou le lien qui s'établit entre un sujet et un objet;

\footnotetext{
${ }^{11}$ Le terme est employé par Pottier (2000 et 2012), mais s'applique chez cet auteur à des phénomènes plus variés que celui analysé ici.
} 


\section{le domaine boulique}

(14) ils sont exigeants ils veulent trop de choses

La modalité boulique est un cas de spécification, intentionnelle et motivée, de la conjonction : vouloir / désirer un objet, c'est tendre vers le lien avec cet objet ;

le domaine délibératif

(15) pourquoi votre grand-père a choisi le euh septième ?

choisir précise que la relation de conjonction entre le sujet et l'objet a fait l'objet d'une détermination délibérée ;

\section{le domaine cognitif}

(16) Ah oui moi j'connais quelques injures en Arabe

Tout objet connu ou appris est un objet que le sujet s'approprie cognitivement.

le domaine de la possession

(17) nous avions acheté un petit appartement au sixième sans ascenseur

acquérir (et ses différents modes, comme acheter), posséder (donc, avoir) sont les procès de la conjonction par excellence ;

\section{le domaine physique}

(18) on porte les robes de sa sœur aînée

Le contact physique constitue la conjonction la plus directe entre le sujet et l'objet ;

\section{le domaine des activités}

(19) en fait il était monté à Paris comme on disait à ce moment là faire ses études

faire ici n'est pas un verbe CHANG ; plutôt, il indique une relation entre le sujet et une activité (étudier)

Il existe une relation contraire à la conjonction : la disjonction, qui caractérise la perte ou la dégradation du rapport de X à Z ; ainsi :

(20) j'ai vendu ma voiture après la la mort de mon mari

(21) Henri est devenu archéologue il est parti en + , mais il venait d' perdre son père

(22) alors moi je déteste les pigeons 
En 20, le propriétaire se retrouve dépossédé de son véhicule ; la conjonction est achevée. De même, en 21 , la conjonction entre Henri et son père est rompue. En 22, il y a disjonction affective (sans qu'il y ait nécessairement eu conjonction auparavant). D'autres verbes, comme oublier quelque chose, quitter (un endroit ou quelqu'un) expriment une conjonction interrompue entre le sujet et l'objet. Le rapport de disjonction est noté $\mathbf{X} \mathbf{V} \mathbf{Z}$.

Enfin, un troisième rapport, qualifié de jonction, est possible. Il s'agit alors de maintenir la conjonction (donc, de ne pas opérer une disjonction). Conserver, garder, maintenir, etc. précisent ce type de relation. Par exemple :

(23) c' qui est très important c'est d'conserver les oui des liens

Le rapport jonctif est noté $\mathbf{X}-\mathbf{Z}$.

Conjonction / disjonction / jonction constituent des relations dont on peut penser qu'elles ont leur origine dans notre expérience la plus fondamentale. En termes cognitifs, il s'agit d'une «image schéma» (Johnson, 1987: 29), fruit de l'interaction des sujets avec leur environnement. Elle résulte de nos actions les plus banales, telles que saisir un objet, et en cela constitue un schéma sensorimoteur - un schème expérientiel - transposable à des domaines très différents.

\subsection{Différentes configurations}

Caractériser la sémantique de NVN avec les seules notions de conjonction/disjonction/jonction se révèle insuffisant; en effet, si ces relations sont toujours orientées du sujet vers l'objet, il existe différentes configurations par lesquelles, et en fonction du verbe et de l'objet concernés, les relations deviennent effectives. Ces configurations impliquent :

- un élément $X$ représentant le référent sujet

- un élément $Z$ représentant le référent objet

- un espace EX représentant l'espace subjectal

- un espace EZ représentant l'espace objectal

- un rapport d'inclusion entre un élément et un espace

- un rapport d'exclusion entre un élément et un espace

La notion d'espace à laquelle nous nous référons n'est pas une réalité préexistante en dehors de l'énoncé ; elle émerge de la relation d'inclusion et d'exclusion que l'on identifie dans toute occurrence de NVN. En effet, selon les cas, NVN peut mettre en jeu l'intégration ou l'exclusion du référent sujet (X) dans l'espace objectal (c'est-à-dire, l'espace de l'objet, EZ), ou bien l'intégration ou l'exclusion du référent objet $(Z)$ dans l'espace du sujet (espace subjectal, EX). Ce jeu de rapports se réalise selon six configurations possibles :

Configuration 1 : Pour $\mathrm{X} \Lambda \mathrm{Z}$, il y a inclusion de l'élément objet $\mathrm{Z}$ dans l'espace subjectal EX. 


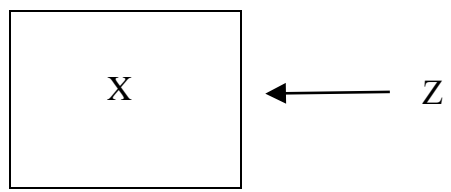

Cette relation est naturellement marquée par avoir ${ }^{12}$, mais un très grand nombre d'autres verbes sont possibles :

(24) le chien devait courir pour l'attraper le plus vite possible

(25) ils gagneront d' l'argent pour eux c'est important

Les procès exprimés par 24 et 25 construisent une relation entre $X$ et $Z$, telle que $Z$ est intégré dans EX ; on pourrait gloser par : une fois attrapé $Z$ est inclus dans la sphère de $X$. Une fois gagné, $Z$ (l'argent) est inclus dans la sphère de $X$. Cette configuration vaut pour les procès bouliques et affectifs, mais aussi pour les procès de perception et de cognition : percevoir, ou connaître, c'est entrer en conjonction avec une altérité, et c'est aussi intégrer cette altérité à son propre espace (que l'on désigne généralement par champ - champ de perception, champ de connaissance).

Configuration 2 : Dans cette configuration où $\mathrm{X} \mathrm{V} \mathrm{Z,} \mathrm{Z} \mathrm{est} \mathrm{en} \mathrm{relation}$ d'exclusion d'avec EX

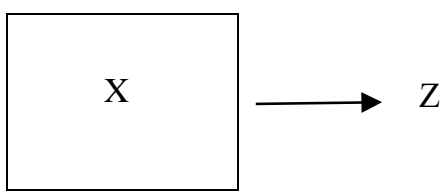

(26) mais c'est d'un coup on les abandonne (les gens)

Ici, Z (les gens) se retrouve exclu de l'espace social EX

(27) C'est difficile d'avoir perdu cet appartement?

L'appartement est un objet de possession qui a été exclu de l'espace (espace de propriété, mais sans doute également espace affectif) du sujet.

Configuration 3 : Dans ce cas, le sujet est en relation de disjonction d'un espace EZ auquel il appartenait.

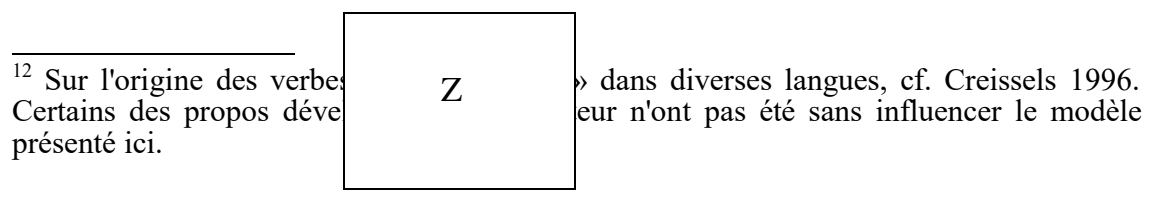




\section{$\mathrm{X}$}

(28) c'était comme de quitter l'ventre de ma mère hein ça c' était totalement freudien

$\mathrm{X}$, qui participait à l'espace «ventre», s'en trouve exclu. Il y a donc disjonction (X V Z) avec EZ. La même configuration serait à l'œuvre dans : il a abandonné la course, le sergent a déserté l'armée, il délaisse sa femme.

Configuration 4 : L'élément $\mathrm{X}$ est ici en conjonction avec EZ $(\mathrm{X} \wedge \mathrm{Z})$

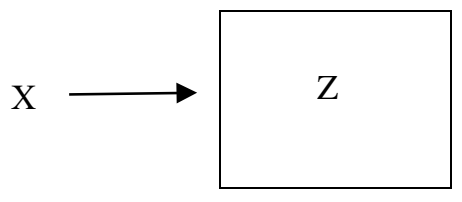

(29) on croise souvent des gens

Quelques exemples, hors corpus : je maintiens ma décision; je conserve mon appétit

Configuration 5 : Ce scénario concerne les cas de jonction : il s'agit pour $\mathrm{X}$ de maintenir $Z$ dans son espace.

(30) est-ce que + vous avez gardé vos amis d'école?

Quelques exemples, hors corpus : je maintiens ma décision; je conserve mon appétit.

Configuration 6 : Ce dernier type de configuration concerne les énoncés jugés très peu transitifs. La conjonction en jeu est d'ailleurs spécifique : elle est de nature «locative» dans la mesure où $\mathrm{X}$ se trouve dans un lieu $\mathrm{Z}$ ou est en coprésence avec un objet $Z$ dont les dimensions peuvent être parcourues ${ }^{13}$. $Z$ est donc configuré comme espace accueillant $\mathrm{X}$. Autre caractéristique, $\mathrm{X}$ est nécessairement en mouvement: il se déplace dans EZ, jusqu'à atteindre les limites de EZ

$\overline{{ }^{13} \text { On lira avec profit le }}$ travail de Sarda (2000) sur les verbes transitifs locatifs. 


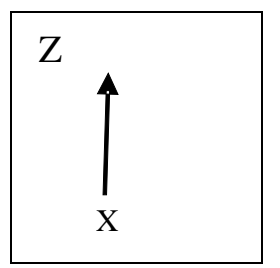

Correspond à cette configuration un énoncé tel que :

(31) le quatre-vingt seize [...] -remonte toute la rue d' Ménilmontant donc c' est un autobus formidable qui traverse tout Paris

La rue Ménilmontant, Paris, sont des espaces géographiques dans lesquels l'autobus (X) est présent, et dans lesquels il se déplace (procès dynamique) jusqu'aux limites de ces espaces. L'espace EZ peut ne pas correspondre à un espace géographique dans des énoncés comme il longe le mur. On le voit, la configuration 6 n'est pas l'inverse de la 5. En effet, X en 6 est nécessairement en mouvement (d'où la flèche). Il semble qu'un énoncé transitif dans lequel $\mathrm{X}$ en tant qu'élément serait maintenu statique dans EZ, est impossible ; existe-t-il un blocage expérientiel ou cognitif à cela?

Une relation de conjonction / jonction / disjonction prend donc place dans une configuration, c'est-à-dire un rapport orienté entre éléments et espaces. Ce rapport est plus complexe que la relation Trajector-Landmark proposée par Langacker (1990), qui nous paraît trop rigide et élémentaire pour appréhender la variété des situations.

\subsection{Application à la transitivité prototypique}

Le modèle sémantique présenté ci-dessus a été illustré par des énoncés syntaxiquement transitifs comprenant des verbes NON-CHANG. Ce même modèle peut-il expliquer les énoncés transitivement prototypiques ? Nous affirmons que oui. Soit cet énoncé avec un verbe (rayer) impliquant un changement d'état de l'objet :

(32) j'en connais quelque chose on vient d'me rayer ma voiture

on peut paraphraser cet énoncé par: l'action (rayer) de X sur Z a causé le changement d'état de $Z$. "L'action de $X$ sur $Z$ » constitue bien un cas de conjonction (en fait, concrètement, l'impact de X sur Z). C'est la configuration 4 qui est pertinente en ce cas, puisque $X$ intègre l'espace $E Z$. En fait, tout énoncé transitif avec verbe CHANG répond toujours à la configuration 4 , même si la représentation de EZ n'est pas aisée à concevoir dans tous les cas. Par exemple,

(33) euh il avait créé une association euh + euh d'aide aux personnes âgées 
l'action de X (ils) portent sur EZ, mais évidemment Z n'est pas référentiellement ou réellement construit en SIT 1; il ne l'est que virtuellement, en tant qu'espace cognitif cible, qui doit prendre forme réelle (SIT 2). L'action de X sur Z constitue bien une conjonction. Comme pour 32, cette conjonction entraîne (ou cause) le changement d'état de Z. On pourrait évoquer le modèle de la boule de billard : la boule de billard blanche propulse en la choquant la boule rouge. Ce choc est la conjonction qui est cause de la propulsion, donc du changement d'état, de la boule rouge. En nous inspirant du schéma de Desclés présenté plus haut, on peut rendre cette représentation par :

Figure 2 - Schéma de la transitivité prototypique

Ainsi, toute causalité présuppose un «contact» entre deux termes, contact qui, dans le cas de la transitivité équivaut à la conjonction de X avec Z. Par ailleurs, la construction transitive a cette particularité, nous semble-t-il, de «montrer» en un seul événement ce qui est nécessairement complexe d'un point de vue causal : la causalité est en principe une relation entre deux termes distincts et autonomes, qui sont, à un moment donné, mis en relation ${ }^{14}$.

Ainsi, notre modèle sémantique de la transitivité s'applique aussi bien à la transitivité non prototypique qu'à la transitivité prototypique ; la conjonction de $\mathrm{X}$ avec $\mathrm{Z}$ est au cœur de la signification de NVN. La différence essentielle entre les énoncés NON-CHANG et les énoncés CHANG est que cette conjonction n'a pas d'effet sur l'objet dans le premier cas, et qu'il en a une dans le second.

\section{DiSCUSSION} ${ }^{14}$ Il faudrait une étude complémentaire pour analyser la particularité des verbes causaux
(causer, provoquer, entraîner...) dans NVN. 
Cette partie a pour objectif d'apporter, sous forme de remarques, des précisions sur certains phénomènes mis en évidence jusqu'à maintenant.

\subsection{Première remarque : configurations et types d'objet}

On observe qu'un même verbe, selon la nature de son objet, répond à des scénarios différents; prenons le cas de la paire abandonner ses enfants / abandonner la course pour laquelle on a X V Z. Dans abandonner ses enfants, l'objet est clairement exclu de l'espace EX du sujet (configuration 2). Dans abandonner la course, le sujet est exclu de l'espace EZ de la course (configuration 3 ). Cette différence est liée à la nature du référent, identique à celle du sujet dans le premier cas (même nature humaine), très différente dans le second où l'objet renvoie à un événement. Autre exemple : rencontrer des problèmes / rencontrer les pêcheurs. Dans le premier syntagme, EX est un espace incluant l'élément Z (rencontrer équivaut à connaître ou à avoir configuration 1) ; dans le second, $\mathrm{X}$ est inclus dans l'espace «pêcheurs» (configuration 4).

\subsection{Deuxième remarque: configuration et synonymie}

Deux verbes synonymes, comme abandonner et quitter peuvent être employés dans des énoncés répondant à des configurations différentes : abandonner ses enfants (donc, exclusion de $\mathrm{Z}$ dans EX) / quitter ses enfants (exclusion de $\mathrm{X}$ dans EZ). Cette différence a été notée dans le Dictionnaire des Synonymes de la Langue Française de Lafaye (1884) ${ }^{15}$ :

on abandonne ce qu'on a...on délaisse au contraire tous ceux dont on se retire.

\subsection{Troisième remarque : conjonction «avoir» et conjonction «être»}

On peut typer la conjonction ou la disjonction de deux façons différentes selon les configurations. Ainsi, lorsqu'on a $\mathrm{X} \Lambda \mathrm{Z}$ (ou X V Z) dans une configuration dans laquelle il y a un rapport entre EX et Z, la conjonction/disjonction est de type AVOIR/NE PAS AVOIR. Par exemple, pour courir un risque, vouloir une bière, prendre un café, connaître une chanson, il s'agit bien d'《avoir» l'objet Z dans son champ d'expérience/perception/connaissance (EX). Et bien sûr de «ne pas avoir» $Z$ pour oublier ses clés, perdre un ami.

Lorsque X $\Lambda \mathrm{Z}$ (ou X V Z) prend sens dans une configuration dans laquelle il y a un rapport entre EZ et X, la conjonction/disjonction est de type «être avec» / «ne pas être avec»; rencontrer les amis, c'est être avec les amis ; de même pour rejoindre la famille. Pour quitter sa femme, la conjonction est de nature «ne pas être avec»».

On peut se demander si cette différence de nature entre deux types de conjonction n'est pas sans rapport avec les remarques de Benveniste (1966) sur

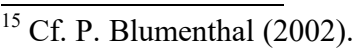


la relation marquée par le verbe avoir et le rapport inverse «être à»? D'une certaine manière, rencontrer ses amis, c'est être $\dot{a}$ ses amis.

\subsection{Quatrième remarque : les constructions à objet pluriel}

Il existe des constructions transitives qui ne répondent pas à la signification dégagée jusqu'à maintenant. Bien loin d'être un problème, ces constructions renforcent et légitiment la notion de conjonction : ce sont les cas où le verbe nécessite obligatoirement un objet pluriel ou deux objets coordonnés. Par exemple

(34) les sorties ça a été important pour souder un petit peu une un collectif d'élèves

(35) elle a toujours comparé les prix

(36) au lieu qu'on essaie de trier les enfants on a fait passer tout le monde

En 34 et 35, la conjonction ne porte pas entre $X$ et $Z$, mais entre les éléments $Z$ : souder, c'est mettre chaque élément en conjonction pour former une seule unité. Comparer, c'est mettre mentalement les éléments en co-présence (conjonction) pour effectuer une évaluation. En 36, trier implique au contraire que l'on «disjoigne» les éléments. A chaque fois, un espace EZ est en jeu (le collectif, l'ensemble des prix, les enfants non triés) et une relation de conjonction / disjonction est présente.

\subsection{Cinquième remarque : les constructions datives trivalentes}

Sans que l'on puisse vraiment développer ici (mais cf. Legallois 2012a et 2012b), le comportement des constructions datives trivalentes N1 V N2 à N3 (elle me prête son stylo, je lui enlève sa robe, je lui prépare une surprise, je lui ai tondu la pelouse, etc.) s'appréhendent encore avec les rapports de conjonction ou de disjonction entre le référent de $\mathrm{N} 3$ et celui de N2. Pour je lui ai tondu la pelouse, énoncé dans lequel le verbe n'est pas a priori trivalent, la conjonction s'établit entre N3 (lui) et un «objet» qui n'est pas la pelouse, mais le résultat de l'action du sujet sur l'objet, soit «la pelouse tondue».

\subsection{Sixième remarque : ambivalence des lectures non causale et causale}

Certains énoncés peuvent être lus de deux manières différentes (cf. plus haut) : soit la lecture (NON-CHANG) relève d'une conjonction «simple» sans effet causal sur le changement de l'objet. Soit une lecture CHANG est possible. Cette ambivalence est liée à la focalisation, présente ou non, sur le changement de situation de l'objet. Ainsi :

(37) par le CROUS on pouvait réserver des courts de tennis 
(38) même pour acheter un cinquante mètres carrés il faudra il faudra qu'ils s'endettent sur cinquante ans enfin ils pourront même pas

Considérer que «des courts de tennis» ou «un cinquante mètres carrés» constituent des objets qui changent d'état par l'action d'un agent est bien sûr possible. Dans ce cas, $X$ entreprend une action sur $Z$ qui va faire que $Z$ va changer d'état. La configuration 4 (X intègre EZ) est en jeu. Mais pour ces énoncés précisément, l'interprétation la plus naturelle n'est pas causale : les énonciateurs se «limitent» à exprimer une relation de conjonction entre un $\mathrm{X}$ et un $\mathrm{Z}$, selon la configuration 1 ( $\mathrm{Z}$ est intégré dans $\mathrm{EX})$ : on pouvait avoir des cours de tennis ${ }^{16} /$ même pour avoir un cinquante mètres carrés... Les verbes réserver et acheter ne font que préciser la conjonction établie par la construction NVN. Ces deux lectures (que l'on a aussi pour La France accumule les dettes «la France a des dettes» / «la France fait qu'une dette vienne en conjonction avec une autre», je bois un café «je prends un café»» ${ }^{17} /$ «j'ingère un café»», etc.) semblent constituer un phénomène régulier, qui montre que la signification invariable de NVN peut, en emploi, varier selon la perspective que l'on adopte sur la relation entre $X$ et $Z$. Cette dernière remarque rejoint l'analyse de Jalenques $(2010: 422)$ sur la différence entre j'évite le sel (disjonction) et on a évité le scandale (on n'a agi sur le scandale «virtuel» ou «possible» pour ne pas qu'il ait lieu).

Cette ambivalence montre que l'analyse syntactico-sémantique ne saurait s'affranchir des circonstances énonciatives.

\section{CONCLUSION}

La tradition grammaticale ainsi que les perspectives cognitives ou fonctionnelles n'ont pas accordé, dans leurs analyses de la transitivité, toute l'attention que NVN méritait. La raison en est simple : les réalisations transitives NON-CHANG ont été perçues a priori comme des cas dégradés, déviants des cas prototypiques, sans que leur homogénéité ait pu être constatée. Au contraire, cet article a voulu entreprendre l'examen de cette transitivité «affaiblie» et a pu montrer que des réalisations apparemment très variables (verbes cognitifs, de perception, d'affectivité, de possession, etc.) partageaient une signification fondamentale : la conjonction du sujet avec l'objet, dans un ensemble de configurations possibles. Les emplois dits prototypiques ne se démarquent que par l'effet de la conjonction sur l'état de l'objet. La perspective adoptée apparaît donc plus rationnelle que celle des tenants de la sémantique du prototypique, puisqu'elle part des emplois ordinaires - les plus fréquents, les moins marqués pour expliquer les emplois plus exceptionnels en usage, ceux qui expriment au plus près l'action prototypique. Mais on ne saurait confondre expression de l'action prototypique et signification linguistique de la construction.

Bien sûr, beaucoup de points restent en suspens, en particulier la sensibilité du passif: certaines configurations favorisent-ils l'alternance de NVN avec le passif? Pour répondre à cette question, un examen sur corpus est nécessaire, qui

${ }_{17}^{16}$ Pouvoir a certainement ici un rôle dans cette lecture.

${ }^{17}$ La valeur de l'article est ici importante. 
permettrait de quantifier la répartition des passifs selon les configurations. Un travail en ce sens est actuellement en cours.

Si la perspective développée ici ne répond sûrement pas à toutes les questions que pose la structure NVN, elle permet cependant d'envisager sous un nouvel angle bien des phénomènes identifiés par la tradition linguistique.

\section{BIBLIOGRAPHIE}

Arnault \& Lancelot, 1973 [1676], Grammaire générale et raisonnée, Hildesheim/New-York, Georg Olms Verlag.

Benveniste E., 1966, Problèmes de linguistique générale 1, Paris, Gallimard.

Blinkenberg A., 1960: Le problème de la transitivité en français moderne: essai syntacto-sémantique, Historisk-filosofiske meddelelser 38:1, Det Kongelige Danske Videnskabernes Selskab, København.

Blumenthal, P., 2003, Le centrage du verbe transitif, Syntaxe et sémantique 1, 4, p. 15-46.

Branca-Rosoff S., Fleury S., Lefeuvre F. et Pires M., 2012, Discours sur la ville. Présentation du Corpus de Français Parlé Parisien des années 2000 (CFPP2000).

Condamines A., 2013, Quand la passion autorise la transitivation d'un circonstanciel de lieu, Journal of French Language Studies 23.p. 335-355

Creissels D., 1996, Remarques sur l'émergence de verbes avoir au cours de l'histoire des langues, Faits de langues 7, p.149-158.

Creissels D., 2013, Schèmes de valence pour les verbes bivalents: esquisse d'une typologie, Séminaire de morphosyntaxe DDL vendredi 3 mai 2013, consulté en nov. 2015 sur http://www.deniscreissels.fr/public/CreisselsVerbes bivalents.pdf

Desclés J.-P., 1990, Langages applicatifs, langues naturelles et cognition, Paris, Hermes.

Desclés J.-P., 1998, Transitivité syntaxique, transitivité sémantique, in A. Rousseau (éd.), La Transitivité, Presses Universitaires du Septemprion, p.162-180.

Francois J., 2003, La prédication verbale et les cadres prédicatifs, Louvain, Paris, Editions Peeters

Givón T., 1984, Syntax: A functional-typological introduction, Amsterdam, Benjamins.

Givon T., 1989, Mind, code and context: Essays in pragmatics, Hillsdale, NJ, Erlbaum.

Hagège C., 2005, De la place réelle de la transitivité, ou la typologie linguistique entre passé et avenir, in G. Lazard \& C. Moyse-Faurie (éds.), Linguistique
typologique, Paris, Presses Universitaires du Septentrion, p. 55-69.

Haspelmath M, à paraître, Transitivity prominence, in A.L. Malchukov \& B. Comrie (eds.), Valency classes in the world's languages, Berlin, De Gruyter Mouton.

Hopper P., \& Thompson S., 1980, Transitivity in Grammar and Discourse, Language 56, p. 251-99.

Ibbotson P, Theakston A., Lieven E. \& Tomasello M., 2012, Semantics of the Transitive Construction: Prototype Effects and Developmental Comparisons, Cognitive Science 36(7), p. 1268-1288. 
Jalenques P., 2010, Un exemple d'interaction morphologie-syntaxe-sémantique : les contraintes sur les procès transitionnels au passif «périphrastique», in Actes du VIIIe Congres International de Linguistique Française, 25-27 septembre 2008, Université d'Oviedo, Liens linguistiques. Etudes sur la combinatoire des composants, Berne, Peter Lang, p. 409-425.

Johnson M., 1987, The Body in the Mind: The Bodily Basis of Meaning, Imagination, and Reason, University of Chicago Press.

Lafaye B., 1869, Dictionnaire des synonymes de la langue française, 3e éd., Paris, Hachette.

Lakoff G., 1977, Linguistic Gestalt, CLS 13, p. 236-287.

Langacker R., 1990, Concept, Image, and Symbol: The Cognitive Basis of Grammar, Berlin/New York, Mouton de Gruyter.

Larjavaara M., 2000, Présence ou absence de l'objet. Limites du possible en français contemporain, Academia Scientiarum Fennica.

Lazard G., 1998, De la transitivité restreinte à la transitivité généralisée, in A. Rousseau (éd.), La transitivité, Lille, Presses du Septentrion, p. 55-84.

Lazard, G., 2008, A propos de La quête des invariants interlangues. La linguistique est-elle une science, Paris, Champion.

Legallois D., 2012a, Pour une sémantique intensionnelle des constructions grammaticales, in M. Van Peteghem, P.Lauwers, E. Tobback, A. Demol et L. De Wilde (éds.), Le verbe en verve, Gent, Academia Press, p.25-46

Legallois D., 2012b, Existe-t-il une énantiosémie grammaticale? Réflexions à partir de la construction dative trivalente, in J. François, P. Larrivée, D. Legallois et F. Neveu (éds.), Linguistique de la contradiction, Berne, Peter Lang.

Naess A., 2007, Prototypical transitivity, Amsterdam, J. Benjamins.

Pottier B., 2000, Représentations mentales et catégorisations linguistiques, Paris-Louvain, Editions Peeters.

Pottier B., 2012, Images et modèles en sémantique, Paris, Éditions Honoré Champion.

Sarda L., 2000, L'expression du déplacement dans la construction transitive directe, Syntaxe et Sémantique 2, p. 121-137.

Stefanowitsch A. \& Gries S., 2003, Collostructions : Investigating the Interaction of Words and Constructions, International Journal of Corpus Linguistics 8, p. $209-243$

Thompson S. \& Hopper P., 2001, Transitivity, clause structure, and argument structure: Evidence from conversation, in J. Bybee \& P. Hopper (eds.), Frequency and the Emergence of Linguistic Structure, Amsterdam/Philadelphia, Benjamins, p.27-56.

Tsunoda T., 1985, Remarks on Transitivity, Journal of linguistics 21, p. 385396.

Vázquez Rozas, V., 2007, A usage-based approach to prototypical transitivity, in N. Delbecque \& B. Cornillie (éds.), Interpreting Construction Schemas From Action and Motion to Transitivity and Causality. Berlin, New York, Mouton de Gruyter, p. 17-38. 